\title{
Physical modeling of pipeline resonance in civil buildings
}

\author{
Elena Yurchenko ${ }^{1, *}$, Konstantin Makarov ${ }^{1}$, Astkhik Kakosian ${ }^{1}$, and Eric Biryukbaev ${ }^{1}$ \\ ${ }^{1}$ Sochi State University, 354000, 94, st. Plastunskaya, Sochi,Russia
}

\begin{abstract}
The method of experimental determination of the magnitudes of pipelines resonant vibrations on small-sized physical models of 1:10 of actual size is shown. The measured values of the oscillation frequencies of pipe models with hinged and pinched supports on both sides are compared with their mathematical counterparts. Fluctuations of pipes with clogging allow us to determine its effect on the values of the resonant frequencies. The obtained results are essential for predicting accidents and fatigue failures of pipelines for various purposes. During the tests on steel models, the excited harmonic sound oscillations of the models are captured by an electromagnetic transducer and transmitted through a microphone amplifier to a computer that serves as an oscilloscope.
\end{abstract}

\section{Introduction}

The pipelines of the building engineering systems operate under the influence of both static and dynamic nature. At the same time, the requirements for the performance and reliability of pipe systems are becoming tougher every year. One of the main factors of reducing the performance of pipelines is vibro-acoustic loads and resonant phenomena. These include vibration loads, fluctuations in pressure and flow rate of the working fluid, leading to depressurization of pipeline connections and the appearance of fatigue cracks. It is precisely the pressure fluctuations, the amplitude of which can reach $50 \%$ of the average value, are one of the reasons leading to vibration, fatigue breakdowns of pipelines.

The uniqueness of the phenomenon of pipelines resonance lies with all its extensive knowledge of the everyday manifestation, especially in the operation of both multi-family and private buildings [1]. This phenomenon is undoubtedly related to the individuality of the engineering systems solutions and encountered errors in the "engineering solutions", which are witnessed by consumers experiencing the discomfort of the sound of vibrating pipelines. According to [2], it is difficult to get rid of systems of resonance oscillations due to their uniqueness, not the reliability of numerical solutions, since for the adequacy of computational models it is necessary to evaluate their individual technical features, physical and operating conditions. Small-sized modeling occupies an insignificant share in the study of resonant phenomena of internal engineering systems of buildings. Basically, they are represented by mathematical models of large-diameter pipelines for engineering structures. Instrumental measurements, including those in emergency conditions, are controlling and

Corresponding author: wsonormalno@yandex.ru 
also are carried out at operating enterprises. Obtaining values of natural oscillations of pipelines with different fixing conditions and their combination, modeling clogging are advantages that are difficult to take into account in mathematical modeling or in kind. The purpose of the simulation is to improve the operational reliability of systems and prevent the effects of vibration exposure. The cheapest way to account for the listed features is to test small-scale models of systems and compare the obtained data with mathematical ones.

The fundamentals of physical modeling laid by Sedov L.I. proceed from such a physical similarity between model and nature, when at similar times and at similar points in space, the values of variables characterizing the behavior of the model are proportional to the values of the corresponding values of nature. As shown in [3-6] a clear difference between the travelling wave and resonance theories is therefore that the peak amplitude is reached before the resonance place in the former and at the resonance place in the latter. Some other effects of resonant vibrations of pipelines are investigated in the works [7-8].

To solve a large number of technical problems, it is of interest to study nonlinear resonance phenomena in mechanical systems when subjected to external periodic loads [9]. For realizing such phenomena, it is necessary to fulfill certain relationships between the natural oscillation frequencies of non-linearly interconnected partial systems or between the natural frequencies and the external excitation frequency. Under these conditions, prerequisites are created for the redistribution of energy between the various generalized coordinates of the system, as a result of which oscillations can be excited in those forms and in directions in which external perturbative loads do not directly act.

An internal property of such oscillatory systems is a stepwise change in their behavior with a continuous change in external conditions [9]. Thus, a string or rod under the action of a vibration load acting in the same plane can perform both flat and spatial oscillations, depending on the values of the parameters of the problem. Qualitatively different stress fields and, accordingly, different strength characteristics are features of various driving modes. Therefore, the actual problem is the creation of mathematical models of nonlinear systems and finding all existing solutions. When small-scale modeling of resonant oscillations at 1:10 of the magnitude of nature, recommendations are used, according to which the product resonance is considered as a phenomenon of increasing the amplitude of forced oscillations twice or more with constant external influence, occurring at frequencies of vibration loads close to the natural frequency products.

In natural engineering systems, according to, the definition of the resonance of pipelines is similar. They are considered oscillations of one end of the pipeline due to relative vibration of the other with a frequency equal to the natural frequency of oscillation, when the amplitude in the middle of the section is dozens of times greater than the amplitude of oscillation of the fixed ends. The most common is rigid fastening. For the numerical analysis of the results of the experiment with small-sized models, we proceed from the provisions of [10], which consider pipeline oscillations with a liquid, where the pipe is considered to be homogeneous, a section in the form of a circle with constant dimensions and the center of inertia coinciding with the axis of the pipe. Neglecting the thickness of the pipe, the system is considered as an elastic string with a linear density consisting of the linear density of the pipe and the fluid flowing inside at a constant velocity without friction. Such a model in [10] was taken from the clause that the diameter of the pipe is much less than its length. This allows the pipe motion equation to be reduced to the equation for a conventional harmonic string. And although the amplitude of oscillations of the tube points at which measurements are made will depend on the position of the source of forced oscillations, the phase difference for two points located at a certain distance from the source will not be. These allowed us to record the oscillogram using one channel during the experiment, having one converter.

According to [11], the oscillogram at resonance will include not only pure sinusoids, but 
also harmonics. The authors [12] argue that the occurrence of resonance, even in the second harmonic, leads to a serious violation of both the integrity of the pipes themselves and their connections [9].

\section{Methods}

The technique of conducting experiments with physical small-sized models of pipelines is based on the conditions outlined in, mathematical modeling based on theoretical substantiations [13]. According to Attachment 6, to indicate the resonance of the structure, devices are used that control the change in the amplitude of oscillations of the resonating part of the tested products relative to the amplitude of vibrations of their attachment points.

In the experimental assembly were tested small-sized models:

model No1 - steel pipe with a diameter of $7.3 \mathrm{~mm}$ with a wall thickness of $0.5 \mathrm{~mm}$;

model No2 - a steel pipe with a diameter of $4.4 \mathrm{~mm}$ with a wall thickness of $0.2 \mathrm{~mm}$.

Models length is $450 \mathrm{~mm}$. The blockage was simulated by placing the wire $0.2 \mathrm{~mm}$ thick along the entire length of the Model Nol pipe. The sizes of models have a scale of 1:10. Geometric characteristics are shown in table 1.

Table 1. Geometric characteristics of models

\begin{tabular}{|c|c|c|c|}
\hline No & $\begin{array}{c}\text { Section/area, } \\
\mathbf{m} / \mathbf{m}^{2}\end{array}$ & $\begin{array}{c}\text { Linear weight, } \\
\text { MN/m }\end{array}$ & $\begin{array}{c}\text { Moment } \\
\text { inertia, } \mathbf{m}^{4}\end{array}$ \\
\hline 1 & $0.0073 \times 0.0005 / 1.14 \times 10^{-5}$ & $8.84 \times 10^{-7}$ & $7.64 \times 10^{-11}$ \\
\hline 2 & $0.0044 \times 0.0002 / 0.276 \times 10^{-5}$ & $2.15 \times 10^{-7}$ & $6.69 \times 10^{-12}$ \\
\hline
\end{tabular}

The scale factor calculated according to for he measured frequency is 10:1. The forced oscillations of the models were created by the player playing mp3 sound recording files with specified frequencies of $24 \mathrm{~Hz}, 28 \mathrm{~Hz}, 70 \mathrm{~Hz}, 150 \mathrm{~Hz}$ independently of each other. Playing sound recordings created sinusoidal oscillations in the form of onedimensional waves with a given frequency.

The oscillations were transmitted to the model by pinching or hinging one end of the pipe on the vibro- isolated acoustic system with the diffuser upward with the magnet down (the oscillation vector is directed along the normal up-down), the other end of the pipe was clamped or hinged on the stand.

Registration of secondary mechanical oscillations of models was carried out using an electromagnetic transducer placed under the middle part of the span of the pipe. The electromagnetic converter was connected to the right channel of the microphone lowfrequency amplifier.

The microphone amplifier was connected with its output to the line-in input of a computer sound card with the SpectraLAB program, which allows the computer to operate in oscilloscope mode. The oscillograms in the SpectraLAB program were used to determine the values of oscillation periods in seconds and amplitudes in $\mathrm{mV}$. The maximum movement of the voice coil of the speaker system is, according to the passport data -3.36 $\mathrm{mm}$. The diffuser experiences a maximum offset at the resonant frequency, which, according to the passport, is $55.25-74.75 \mathrm{~Hz}$. The movement is due to electromagnetic forces and the length of the winding wire on the voice coil. The coil in the diffuser is made of copper enameled wire with a diameter of $0.140 \mathrm{~mm}$, the winding is double-layered, in the first layer 24 turns, in the second - 21 turns, the height of the winding is $3.9 \mathrm{~mm}$, and the DC resistance is $3.9-4.0 \mathrm{Om}$.

The electromagnetic converter was located under the middle of the span of the model. Its length is $20 \mathrm{~cm}$, so it caught vibrations at distances from supports on a quarter of a span. 
The converter consists of an inductance coil wound with copper enameled wire with a DC resistance of $54 \mathrm{Om}$ and a ferromagnetic core assembled from rectangular ferrite plates glued together and two aluminum pole pieces. The principle of operation of the converter is based on the registration of EMF induction in the coil of an electromagnetic transducer in a variable magnetic field of an oscillating sample made of a ferromagnetic material (steel) arising from the interaction of the magnetic field of the sample with the transducer.

All of the above equipment had an autonomous DC power supply to minimize electrical interference (Figure 1).

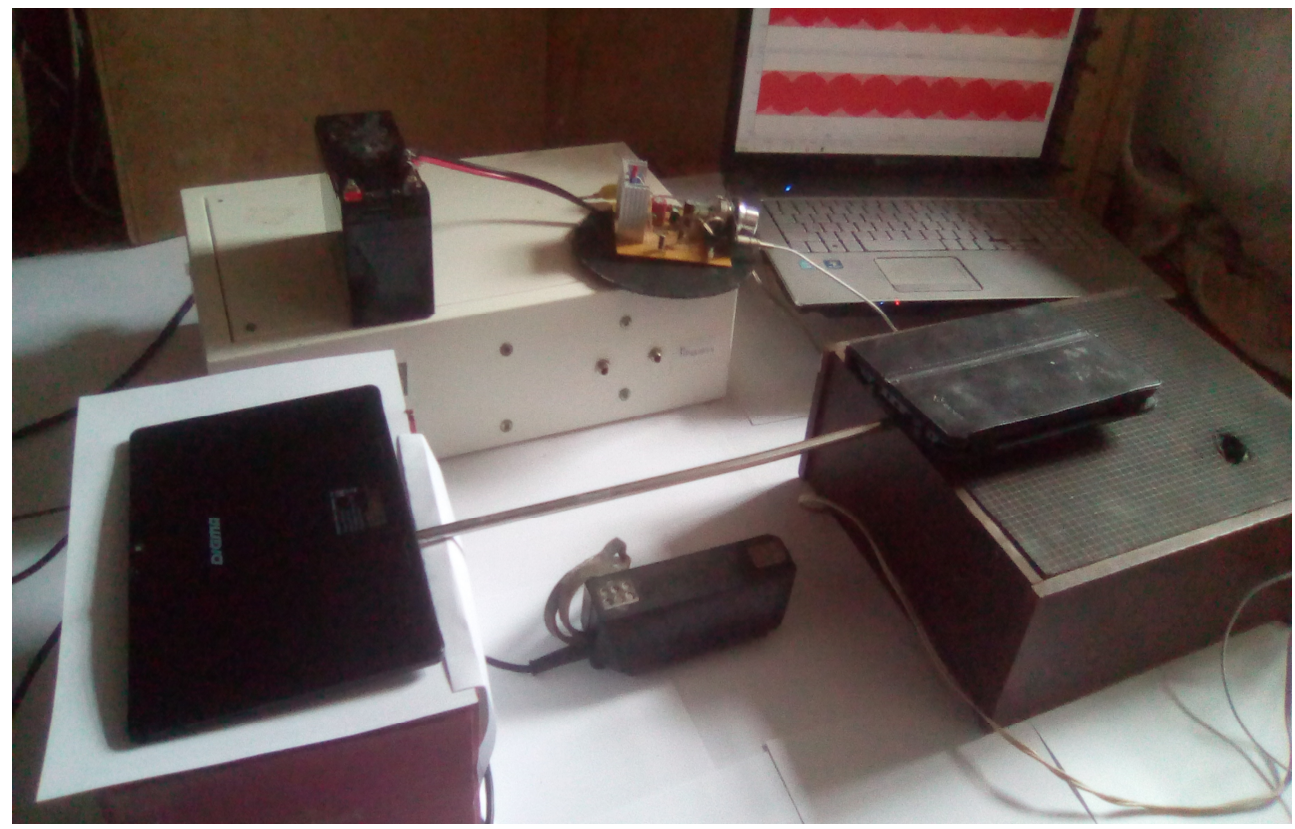

Fig. 1. Model test pattern

The mathematical model provided for the determination of the natural frequencies of the pipe and pipe oscillations with clogging as for a beam on two supports with hinges and pinching from two sides, pinching on one side with the mass of the model itself, distributed along the length. The numerical values for the first three oscillation frequencies are determined according to the method [14]:

$$
f_{01}=\left(\lambda_{\mathrm{i}}^{2} / 2 \pi \mathrm{l}^{2}\right) \sqrt{ }\left(\mathrm{E} \mathrm{l}_{\mathrm{y}} / \mathrm{Pf}\right)
$$

Where

$E$ - Elastic modulus;

$j$ - Imaginary unit indicating that the force vectors are shifted by an angle $\pi / 2$;

$J$ - Moment of inertia of the section;

$\rho$ - density;

$\lambda_{i}$ - frequency coefficient according to table 3.1 , taken depending on the model fixing scheme and number

$\mathrm{i}$ - form of frequency.

By this definition:

-for a pipe with a diameter of $0.0073 \mathrm{~m}$ with one side clamping $-f_{1}=11.8 \mathrm{~Hz}$;

$f_{2}=73.2 \mathrm{~Hz} ; f_{3}=205.2 \mathrm{~Hz}$;

-For pipes with a diameter of $0.0073 \mathrm{~m}$ with hinges on both sides $-f_{1}=32.9 \mathrm{~Hz}$;

$f_{2}=131.6 \mathrm{~Hz} ; f_{3}=296.8 \mathrm{~Hz}$; 
-For a pipe with a diameter of $0.0073 \mathrm{~m}$ when pinched $-f_{1}=74.5 \mathrm{~Hz} ; f_{2}=205.2 \mathrm{~Hz}$;

$f_{3}=402.9 \mathrm{~Hz}$

-For a pipe with a diameter of $0.0044 \mathrm{~m}$ when pinched on one side $-f_{1}=7.1 \mathrm{~Hz}$;

$f_{2}=44.1 \mathrm{~Hz} ; f_{3}=123.8 \mathrm{~Hz}$;

-For a pipe with a diameter of $0.0044 \mathrm{~m}$ with hinges on both sides $-f_{l}=19.8 \mathrm{~Hz}$;

$f_{2}=79.2 \mathrm{~Hz} ; f_{3}=124.0 \mathrm{~Hz}$;

-For pipe with a diameter of $0.0044 \mathrm{~m}$ with clamping on both sides $-f_{1}=44.9 \mathrm{~Hz}$; $f_{2}=123.7 \mathrm{~Hz} ; f_{3}=242.6 \mathrm{~Hz}$.

The ratio between the first resonant frequencies for the models is shown in Figure 2. The ratio between the first resonant frequencies for the models is shown in Figure 2. As we can see, the smaller diameter of the pipes, the greater the value of the natural frequency.

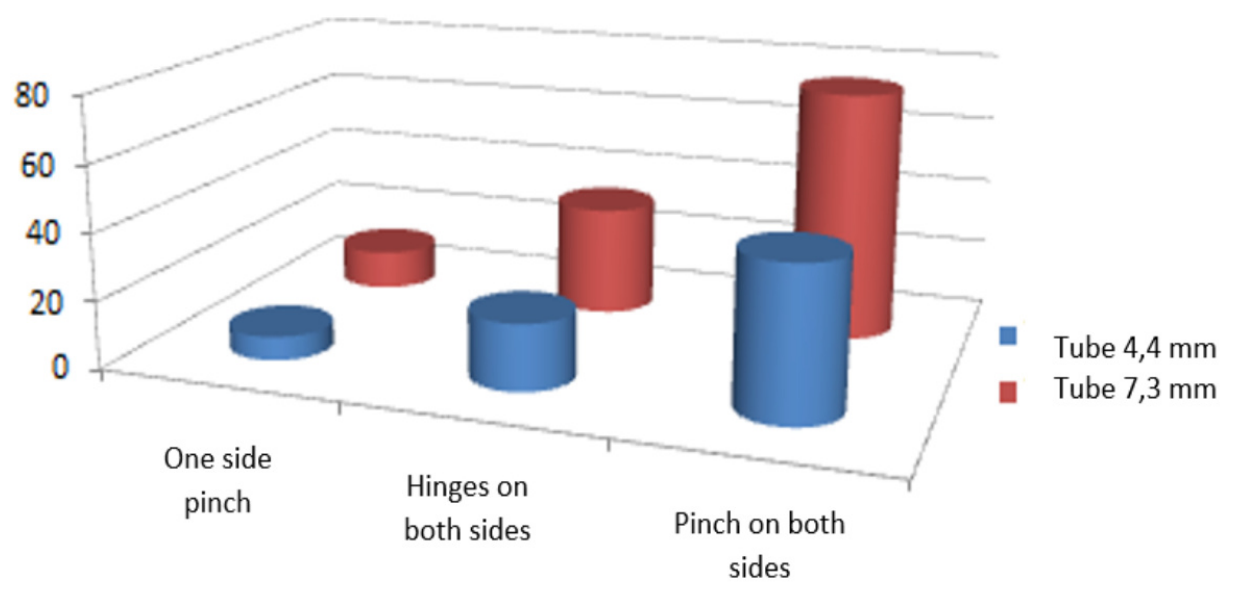

Fig. 2. The relationship between the first resonant frequencies for models

The amplitudes of vibration accelerations are determined according to the method [15]. Since the diffuser experiences a maximum displacement of $c=3.36 \mathrm{~mm}$ at the resonant frequency of $\mathrm{f}_{\text {res. }}$. Which, according to the passport, is $55.25-74.75 \mathrm{~Hz}$, the maximum speed $v$ of such a vibration will be equal to: $v=0.185(0.252) \mathrm{m} / \mathrm{s}$, the acceleration a is equal to the offset c multiplied by the square of the frequency $f_{\text {res }}: a=c f_{\text {res }}^{2}=10.16(18.9) \mathrm{m} / \mathrm{s}^{2}$, what is $1.03 \mathrm{~g}(1.92 \mathrm{~g})$.

The amplitude of the vibration acceleration of the model is calculated at a model point with a coordinate

$\xi=0.5 \times l$ ( $l$ is the model span) on two sides at a forced frequency of $70 \mathrm{~Hz}$, coinciding with the resonant frequency of the diffuser $55.25-74.75 \mathrm{~Hz}$ and the measured frequency. Mechanical loss factor:

$$
\eta_{1}=1 /\left(f_{1}\right)^{0,5}=0,12
$$

According to [16] the recommended values of $\eta$ generally lie within the following limits by $0.01<\eta<0.1$. From [15] for the relative coordinate $\xi=x / l=0.5$ we choose the coefficient of oscillation shapes $K i(\xi)$, depending on the model fixing scheme, the coordinates of the point where the oscillation shape is determined. $K_{l}(0.5)=1.31$. According to [15] transfer coefficient:

$$
\mu_{1}=\sqrt{ }\left[\left(\mathrm{K}_{1}^{2}(\zeta) / \mathrm{n}_{1}^{2}\right)+1\right]
$$


$\mu_{l}(0.5)=10.95$, the acceleration at the point with coordinate $\xi$ will be: $Z=\mu_{1} a, Z(0.5)=11.27 g$ (21.02 g).Computer simulation was performed in the software complex Lyra 2017. Modeling a blockage program in contrast to that used in Aspen HYSYS V8.0 [16-19], the aging of the pipeline in the form of corrosion was not consider. The effect of pipe wall thickness with a uniform diameter was investigated in [14] by numerical simulation in the ANSYS complex. It is shown that with an increase in the thickness of the pipe wall, the values of natural frequencies decrease.

The pipeline is presented in the form of a beam with a length of $0.45 \mathrm{~m}$, clamped on one side, loaded with its own weight along the entire length. A harmonic sinusoidal load without additional mass with an amplitude equal to the weight of the beam, a phase shift equal to zero is applied on the side of the non-grounded end of the beam. The oscillation parameters corresponded to the forced frequencies in the physical simulation and were accordingly: $24 \mathrm{~Hz}-150 \mathrm{rad} / \mathrm{s}, 28 \mathrm{~Hz}-175 \mathrm{rad} / \mathrm{s}, 70 \mathrm{~Hz}-439 \mathrm{rad} / \mathrm{s}, 150 \mathrm{~Hz}-942 \mathrm{rad} / \mathrm{s}$. According to the results of calculations, the natural frequency of oscillations of a pipe with a diameter of $7.3 \times 0.5 \mathrm{~mm}$ is $16 \mathrm{~Hz}$, and pipes with a diameter of $4.4 \times 0.2 \mathrm{~mm}$ is $5 \mathrm{~Hz}$. Thus, the displacement results for a pipe with a diameter of $7.3 \times 0.5 \mathrm{~mm}$ are close to the natural frequency. At a distance from the clamped support to $1 / 4-1 / 2$ of the span of movement, they amount to - 2.09-7.76 mm (Figure 3).

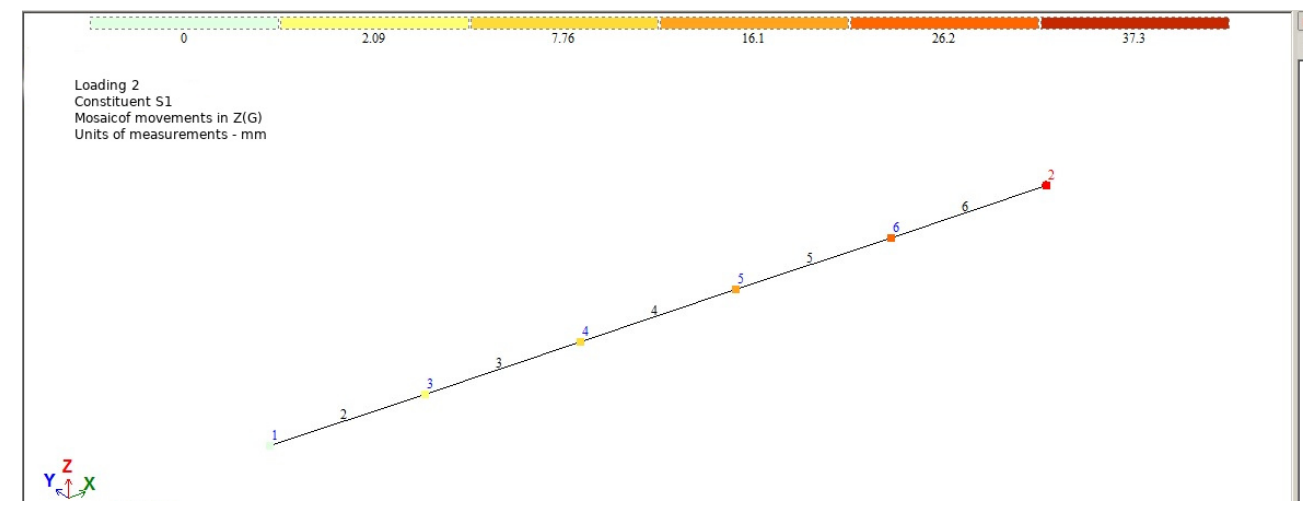

Fig 3. Movement of model points with a harmonic effect on a pipe with a section of $7.3 \times 0.5 \mathrm{~mm}$

\section{Results and Discussion}

Oscillograms were obtained at forced frequencies of 24, 28, 70 and $150 \mathrm{~Hz}$, the measured frequencies are presented in Table 2 and Figure 4. Those measured frequencies of the models that had values close to the stimulated and with the largest oscillation amplitudes were considered to be resonant. They consider displacements at a forced frequency of 70 $\mathrm{Hz}$, since the displacements of both models with a cross section of $7.3 \times 0.5 \mathrm{~mm}$ and $4.4 \times 0.2$ $\mathrm{mm}$ in the middle of the span are the largest. As can be seen from the mathematical model, the frequency of $70 \mathrm{~Hz}$ is the first resonant for a pipe with a cross section of $7.3 \times 0.5 \mathrm{~mm}$ with pinching from two sides and the second resonant with pinching from one side. For a pipe with a cross section of $4.4 \times 0.2 \mathrm{~mm}$, this frequency is the second resonance at the hinges. 
Table 2. Results of testing pipe models

\begin{tabular}{|c|c|c|c|c|}
\hline \multirow[b]{2}{*}{$\begin{array}{c}\text { Forced } \\
\text { frequency } \mathrm{Hz}\end{array}$} & $\begin{array}{c}\text { Amplitude, } \\
\text { mm }\end{array}$ & $\begin{array}{c}\text { Measured } \\
\text { frequency, } \mathbf{H z}\end{array}$ & $\begin{array}{c}\text { Amplitude, } \\
\text { mm }\end{array}$ & $\begin{array}{c}\text { Measured } \\
\text { frequency, } \mathrm{Hz}\end{array}$ \\
\hline & \multicolumn{2}{|c|}{$\begin{array}{l}\text { Pipe section } 7.3 \times 0.5 \mathrm{~mm} \text { with } \\
\text { pinching on one side and } \\
\text { conditional pinching on the side } \\
\text { of the diffuser }\end{array}$} & \multicolumn{2}{|c|}{$\begin{array}{l}\text { Pipe section } 4.4 \times 0.2 \mathrm{~mm} \text { with } \\
\text { pinching on one side and } \\
\text { conditional pinching on the side } \\
\text { of the diffuser }\end{array}$} \\
\hline $24 \mathrm{~Hz}$ & 2.69 & 23 & 2.78 & 25 \\
\hline $28 \mathrm{~Hz}$ & 2.45 & 27 & 2.98 & 27 \\
\hline $70 \mathrm{~Hz}$ & 3.04 & 71 & 3.28 & 67 \\
\hline $150 \mathrm{~Hz}$ & 2.92 & 166 & 3.2 & 142 \\
\hline $\begin{array}{l}150 \mathrm{~Hz} \text { with } \\
\text { blockage }\end{array}$ & 2.79 & 125 & & \\
\hline
\end{tabular}

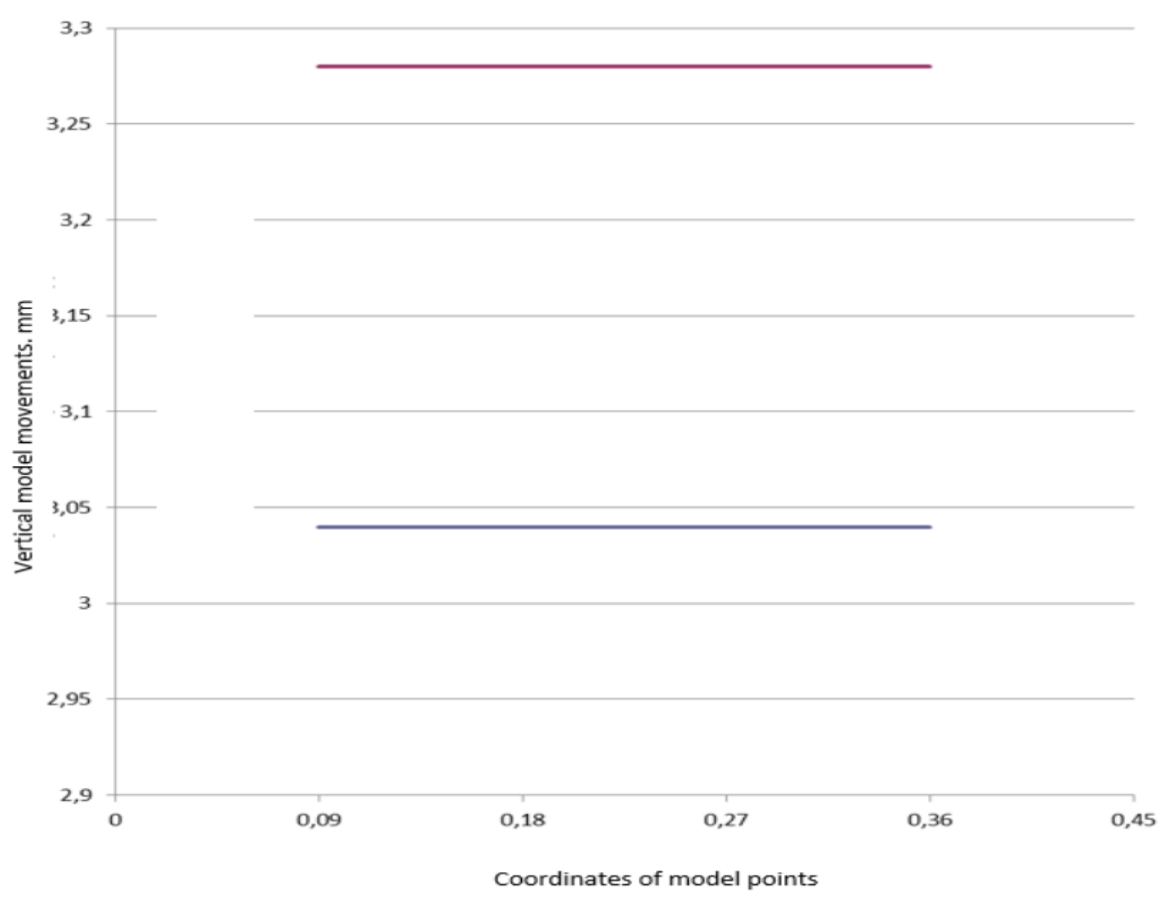

Fig. 4. Moving points of pipe models $7.3 \times 0.5 \mathrm{~mm}$ (blue line color), pipe models $4.4 \times 0.2 \mathrm{~mm}$ (green line color) at a forced frequency of $70 \mathrm{~Hz}$

\section{Conclusions}

1. The test results of a physical small-size pipeline model are confirmed on a mathematical model and allow you to set the mode of resonant oscillations at a forced frequency.

2. A blockage in the form of a wire with a diameter of $0.2 \mathrm{~mm}$ inserted into the test tube played the role of vibration isolation, which is often observed in practice. 
3. It is possible to take into account the study of the uniqueness of the engineering system in the physical modeling on a small-sized model, which ensures a low cost of testing and is sufficient for solving engineering problems.

\section{References}

1. V.V. Aleksashina, Earthquake engineering. Safety of facilities (2007)

2. A.A.Vodka, D.K.Tihij, A.I.Trubaev, Ju.N Ul'janov. Studies of the dynamic characteristics of the pipeline under laboratory conditions (2014)

3. A. A. Bell, Resonance Approach to Cochlear Mechanics (2012)

4. R. Patuzzi, Cochlear Micromechanics and Macromechanics (2010)

5. H. Duifhuis, Cochlear Mechanics: Introduction to a Time Domain Analysis of the Nonlinear (2010)

6. K. Gomez, PACE (2013)

7. A.I. Municyn, Diss. of doctor of technical Sciences (2011)

8. A.P. Medvedev, V. G. Lebedev, Bull. Udm. University 4 (2006)

9. M.L. Kagan, D.Ju. Gustov. Vestnik MGSU, 4 (2011)

10. A. Prokofiev, G. Makariyants, E. Shakhmatov, 17th International Congress on Sound and Vibration (ICSV17). (Cairo, Egypt, 2010)

11. A. Gulbraar, Billington Process Technology at AspenTech OPTIMIZE TM (Washington. D.C. 2011)

12. A. Jones, Genesis Oil and Gas Consultants at Aspen Tech OPTIMIZE 2011 Conference Modeling (Washington. D.C. 2011)

13. Y.I. Kolesnikov, K.V. Fedin, A.A. Kargapolov Near Surface Geoscience 2012 - 18th European Meeting of Environmental and Engineering Geophysics (Paris. France, 2010)

14. L.G. Pastuhova, A.A. Sekacheva., Int. J. Comp. Civ. Str. Eng., 4 (2016) 